\title{
Combined analysis of AFP and HCCR-1 as an useful serological marker for small hepatocellular carcinoma: A prospective cohort study
}

\author{
Guoxin Zhang ${ }^{\mathrm{a}}$, Seon-Ah Ha ${ }^{\mathrm{b}}$, Hyun K. Kim ${ }^{\mathrm{b}}$, Jinah Yoo $^{\mathrm{b}}$, Sanghee Kim ${ }^{\mathrm{b}}$, Youn S. Lee ${ }^{\mathrm{c}}$, Soo Y. Hur ${ }^{\mathrm{d}}$, \\ Yong W. Kim ${ }^{\mathrm{d}}$, Tae E. Kim ${ }^{\mathrm{d}}$, Yong G. Park ${ }^{\mathrm{e}}$, Jing Wang ${ }^{\mathrm{a}}$, Yang Yang ${ }^{\mathrm{a}}$, Zekuan Xu ${ }^{\mathrm{a}}$, Eun Y. Song ${ }^{\mathrm{f}}$, \\ Zuhu Huang ${ }^{\mathrm{a}}$, Peng Jirun ${ }^{\mathrm{g}}$, Jin Zhongtian ${ }^{\mathrm{g}}$, Qiao Shishi ${ }^{\mathrm{g}}$, Cui Zhuqingqing ${ }^{\mathrm{g}}$, Gong Lei ${ }^{\mathrm{g}}$ and \\ Jin W. Kim ${ }^{\mathrm{b}, \mathrm{d}, *}$ \\ ${ }^{a}$ Department of Gastroenterology, the First Affiliated Hospital of Nanjing Medical University, Nanjing, China \\ ${ }^{\mathrm{b}}$ Department of Molecular Genetic Laboratory, College of Medicine, the Catholic University of Korea, Seoul, \\ Korea \\ ${ }^{\mathrm{c}}$ Department of Clinical Pathology, College of Medicine, the Catholic University of Korea, Seoul, Korea \\ ${ }^{\mathrm{d}}$ Department of Obstetrics and Gynecology, College of Medicine, the Catholic University of Korea, Seoul, Korea \\ ${ }^{\mathrm{e}}$ Department of Biostatistics, College of Medicine, the Catholic University of Korea, Seoul, Korea \\ ${ }^{\mathrm{f}}$ The Korea Research Institute of Bioscience and Biotechnology, Daejeon, Korea \\ ${ }^{\mathrm{g}}$ Department of Hepatobiliary, Surgery, Peking University People's Hospital, Beijing, China
}

\begin{abstract}
Hepatocellular carcinoma (HCC) is one of the most frequent malignant tumors in the world. The only serological marker widely used for the diagnosis of HCC is alpha-fetoprotein (AFP). Despite that AFP is widely used for the diagnosis of $\mathrm{HCC}$, it has a limit as a serological marker due to its low sensitivity and specificity. The human cervical cancer proto-oncogene 1 (HCCR-1) was previously reported as a new biomarker for HCC. To further evaluate the HCCR-1 as a biomarker for HCC, we conducted the prospective cohort study. We evaluated the significance of simultaneous measurement of 2 tumor markers in the diagnosis of HCC in China, Japan and Korea. Two markers for HCC, AFP and HCCR-1, were measured in the sera obtained from 1,338 patients at the time of initial diagnosis of HCC. Of the $1338 \mathrm{HCC}$ patients, $616(46 \%)$ and $686(51.3 \%)$ were sero-positive for AFP and HCCR-1, respectively. The positive rate for HCC was increased up to $74.1 \%$ in combined use of AFP and HCCR-1. Many cases (54\%) for AFP-negative HCC were positive for HCCR-1 and vice versa. More importantly, the diagnostic rate for small HCC $(<2 \mathrm{~cm})$ was significantly improved in the combined analysis of AFP and HCCR-1 to 56.9\% although it was only $40.1 \%$ and $23.4 \%$ in the single analysis of HCCR-1 and AFP, respectively. Our result suggests that the HCCR-1 could be an useful biomarker for HCC while the diagnostic rate could be significantly improved in the combined use of HCCR-1 and AFP.
\end{abstract}

Keywords: Hepatocellular carcinoma, biomarker, AFP, HCCR-1

\section{Abbreviations \\ HCC, hepatocellular carcinoma \\ $\mathrm{CH}, \quad$ chronic hepatitis \\ LC, liver cirrhosis}

HCCR1, human cervical cancer oncogene 1

AFP, alpha fetoprotein

AFPL3, sugar chain of Lens culinaris

DCP, des- $\gamma$-carboxy prothrombin
${ }^{*}$ Corresponding author: Prof. Jin Woo Kim, Molecular Genetic Laboratory, College of Medicine, The Catholic University of Korea, Seoul 137-040, Korea. Tel.: +82 119014 2389; Fax: +82 2593 2389; E-mail: jinwoo@catholic.ac.kr.

\section{Introduction}

Hepatocellular carcinoma (HCC) is the fifth most common cancer in the world and the third leading cause 
of cancer-related death [1]. HCC is the second and the third most common causes of cancer death in China and Korea, respectively [1,2]. Currently, the only available tools for HCC surveillance are testing for serum AFP level and hepatic ultrasonography [3,4]. However, AFP level has poor sensitivity and specificity for HCC [3] and ultrasonography is operator dependent and limited in its ability to discriminate HCC from such nonneoplastic lesions as regenerative nodules [4]. Newer methods for the early detection of HCC are needed in the form of either biomarkers or radiological tests. Although serum AFP level is an useful tumor marker for the detection and monitoring of HCC, the falsenegative rate with AFP level alone may be as high as $40 \%$ for patients with early stage HCC [5]. Even in patients with advanced HCC, the AFP levels may remain normal in $15 \%-30 \%$ of the patients. Other HCC markers, such as des- $\gamma$-carboxy prothrombin (DCP), alkaline phosphatase isoenzyme-I, and tissue polypeptide specific antigen, have been developed to improve the sensitivity, specificity, early detection, and prediction of prognosis; but the overall results have been unsatisfactory [6]. In recent years, clinically useful tumor markers for HCC diagnosis have included AFP, a fucosylated variant of the AFP glycoprotein which has a high affinity to the sugar chain of Lens culinaris (AFPL3), and DCP.

Several laboratories have recently reported that HCCR-1 is expressed by a large proportion of HCCs, but is undetectable in normal hepatocytes and nonmalignant liver disease [2,7-10]. Elevated level of HCCR-1 has been shown to be associated with an increase in the risk of developing HCC [7]. We identified a new oncogene associated with human cervical cancer that is also overexpressed in various human tumors including liver cancer $[7,11,12]$. The functional roles of these oncogenes in tumorigenesis is manifested as a negative regulator of the p53 tumor suppressor [11,12]. HCCR-1 is a $42 \mathrm{kDa}$ protein which is overexpressed in various human tumors including liver cancer $[7,11$, 12]. Northern and Western blot analyses and immunohistochemical studies indicate that the HCCR-1 mRNA and protein was overexpressed in tumorous compared with non-tumorous cirrhotic tissue of HCC [7]. These results indicate that $H C C R-1$ probably represents a new oncogene that is related to liver tumorigenesis, functioning as a negative regulator of the p53 tumor suppressor $[11,12]$. Moreover, the expression of HCCR-1 is modulated by the phosphatidylinositol 3-kinase/Akt pathway which is involved in the tumorigenesis by regulating cell cycle progression and survival [13].
In this study, we undertook the following aims: 1) To investigate the diagnostic efficiency of HCCR-1 in HCC, 2) To investigate its usefulness in the early diagnosis for small HCC, 3) To compare with AFP test, 4) To test for the combined analysis of HCC with AFP and HCCR-1 especially in small HCCs.

\section{Methods and materials}

\subsection{Patients and study design}

1380 subjects were recruited during the study period from September 2003 to August 2007 from 4 clinical sites (Catholic University, Korea; Seoul National University, Korea; Nanjing University, China and Sanyo Chemical, Japan) (Table 1). The cohort consisted of 1006 males (76.1\%) with an average age of 54.4 years and 332 females $(23.9 \%)$ with an average age of 56.6 years (Table 2). Blood samples from Catholic University, Korea and Seoul National University, Korea were frozen for analysis and shipped to our institution. On the contrary, blood samples from Nanjing University, China and Sanyo Chemical, Japan were analyzed at their own laboratory and sent us the results. Clinical data were collected for serum chemistries, imaging (CT, MRI and/or ultrasound), presenting symptoms, medications/interventions/therapeutics, and other medical informations including the presence of hepatitis (Table 2).

The enrollment criteria are as follows. Inclusion criteria are 1) patients were confirmed to have $\mathrm{HBV}$ or HCV infection at study onset, 2) HCC diagnosis histologically confirmed by the examination of liver specimens obtained by percutaneous biopsies, 3) pathological grading was contextually assessed in all the patients according to Edmonson's classification, and 4) patient's informed consent to provide blood samples for AFP and HCCR-1 determination. Exclusion criteria are 1) incomplete medical records, 2) misdiagnosis, 3) follow-up visit cannot be achieved within 30 months period, and 4) previous use of immunomodulators such as interferon. The clinical study consisted of consecutive patients who met both inclusion and exclusion criteria. Because time is irrelevant to our determining the HCC progression, we cannot state success criteria. Rather, we simply measure the HCCR-1 value at the time of visit.

At the end of the study, all evaluable subjects were subdivided into 4 categories by the site investigators based on biopsy, explanted liver histology, imaging 
Table 1

Patient profiles of HCC regarding clinical site information

\begin{tabular}{|c|c|c|c|c|c|c|}
\hline \multicolumn{3}{|l|}{ Site } & \# Subjects & $\%$ Total & \multicolumn{2}{|c|}{ Study period } \\
\hline \multicolumn{3}{|c|}{ Catholic Univ. Hospital, Seoul, Korea } & 686 & 50 & \multicolumn{2}{|c|}{$9 / 1 / 2003-5 / 30 / 2006$} \\
\hline \multicolumn{3}{|c|}{ Seoul National Univ. Hospital, Seoul, Korea } & 72 & 6 & \multicolumn{2}{|c|}{$10 / 1 / 2004-12 / 30 / 2005$} \\
\hline \multicolumn{3}{|c|}{ Nanjing Univ. Hospital, Nanjing, China } & 380 & 29 & \multicolumn{2}{|c|}{$3 / 1 / 2004-8 / 1 / 2006$} \\
\hline \multicolumn{3}{|c|}{ Sanyo Chemical, Japan } & 200 & 15 & \multicolumn{2}{|c|}{$7 / 1 / 2006-8 / 1 / 2007$} \\
\hline Total & & & 1338 & 100 & & \\
\hline \multicolumn{7}{|l|}{ Site distribution } \\
\hline \multirow[t]{2}{*}{ Site } & Group 1 & Grou & & Group 4 & Group 3 & \multirow[t]{2}{*}{ Total } \\
\hline & $\mathrm{HCC}$ & $\mathrm{CH} / \mathrm{LC} t$ & $\mathrm{HCC}$ & No HCC & Suspicious & \\
\hline Catholic, Korea & 325 & 16 & & 183 & 9 & 686 \\
\hline Seoul, Korea & 65 & 5 & & 0 & 2 & 72 \\
\hline Nanjing, China & 370 & 0 & & 0 & 10 & 380 \\
\hline Sanyo, Japan & 200 & 0 & & 0 & 0 & 200 \\
\hline Total & 960 & 174 & & 183 & 21 & 1338 \\
\hline
\end{tabular}

Table 2

Patient profiles of HCC regarding gender distribution, age distribution, hepatitis status and HCCR-1 values

\begin{tabular}{|c|c|c|c|c|c|c|c|c|c|c|}
\hline \multirow{2}{*}{\multicolumn{3}{|c|}{ Gender distribution }} & \multirow{2}{*}{\multicolumn{2}{|c|}{$\frac{\text { Group } 1}{\text { HCC }}$}} & \multirow{2}{*}{\multicolumn{2}{|c|}{$\begin{array}{l}\text { Group } 2 \\
\mathrm{CH} / \mathrm{LC} \rightarrow \mathrm{HCC}\end{array}$}} & \multirow{2}{*}{\multicolumn{2}{|c|}{$\begin{array}{l}\text { Group } 4 \\
\text { No HCC }\end{array}$}} & \multirow{2}{*}{$\begin{array}{l}\text { Group } 3 \\
\text { Suspicious } \\
\end{array}$} & \multirow[t]{2}{*}{ Total } \\
\hline & & & & & & & & & & \\
\hline \multirow[t]{2}{*}{ Male } & \multicolumn{2}{|c|}{$\mathrm{N}$} & \multicolumn{2}{|l|}{725} & \multicolumn{2}{|c|}{131} & \multicolumn{2}{|c|}{133} & 17 & 1006 \\
\hline & \multicolumn{2}{|c|}{$\%$} & \multicolumn{2}{|l|}{75.5} & \multicolumn{2}{|l|}{75.3} & \multicolumn{2}{|l|}{72.7} & 81.0 & 76.1 \\
\hline \multirow[t]{2}{*}{ Female } & \multicolumn{2}{|c|}{$\mathrm{N}$} & \multicolumn{2}{|l|}{235} & \multicolumn{2}{|l|}{43} & & 4 & 332 \\
\hline & \multicolumn{2}{|c|}{$\%$} & \multicolumn{2}{|l|}{24.5} & \multicolumn{2}{|l|}{24.7} & \multicolumn{2}{|l|}{27.3} & 19.0 & 23.9 \\
\hline Total & & & 960 & & 174 & & 183 & & 21 & 1338 \\
\hline Age dist & bution & & Grol & & Group 2 & & Group & & Group 3 & Total \\
\hline & & & $\mathrm{HCC}$ & & $\mathrm{CH} / \mathrm{LC} \rightarrow \mathrm{H}$ & $\mathrm{CC}$ & No $\mathrm{HC}$ & & Suspicious & \\
\hline Male & $\mathrm{N}$ & & 725 & & 131 & & 133 & & 17 & 1006 \\
\hline & Aver: & $\pm \mathrm{SD}$ & 55.2 & $=6.6$ & $52.4 \pm 5$ & & $51.7 \pm$ & & $54.2 \pm 3.1$ & $54.4 \pm 6.3$ \\
\hline & Rang & & $41-7$ & & $41-64$ & & $41-63$ & & $50-59$ & $41-71$ \\
\hline Female & $\mathrm{N}$ & & 235 & & 43 & & 50 & & 4 & 332 \\
\hline & Aver & $\pm \mathrm{SD}$ & 58.6 & $=4.9$ & $51.8 \pm 5.6$ & & $50.8 \pm$ & & $55.0 \pm 0.0$ & $56.5 \pm 6.0$ \\
\hline & Rang & & $46-7$ & & $40-63$ & & $41-63$ & & $55-55$ & $40-71$ \\
\hline Total & $\mathrm{N}$ & & 960 & & 174 & & 183 & & 21 & 1338 \\
\hline & Aver: & $+\mathrm{SD}$ & 56.0 & $=6.4$ & $52.2 \pm 5.2$ & & $51.5 \pm$ & & $54.3 \pm 2.8$ & $54.9 \pm 6.3$ \\
\hline & Rang & & $41-7$ & & $40-64$ & & $41-63$ & & $50-59$ & $40-71$ \\
\hline Hepatitis & status & Group & & Grol & Ip 2 & Gro & & & oup 3 & Total \\
\hline & & $\mathrm{HCC}$ & & $\mathrm{CH} /$ & $\mathrm{LC} \rightarrow \mathrm{HCC}$ & No & & & picious & \\
\hline $\mathrm{HBV}+$ & & $741(7$ & $7.2 \%)$ & 134 & $(77.0 \%)$ & 137 & $4.9 \%)$ & & $(71.4 \%)$ & $1027(76.8 \%)$ \\
\hline $\mathrm{HCV}+$ & & $186(1$ & $9.4 \%)$ & 28( & $16.1 \%)$ & 33 & $.0 \%)$ & & $23.8 \%)$ & $252(18.8 \%)$ \\
\hline $\mathrm{HBV}+/$ & $\mathrm{ICV}+$ & $33(3$. & $4 \%)$ & 12( & $5.9 \%)$ & 13( & $1 \%)$ & & $4.8 \%)$ & $59(4.4 \%)$ \\
\hline Total & & 960 & & 174 & & 183 & & 21 & & 1338 \\
\hline Distribut & on of $\mathrm{H}$ & CR-1 v & alues $(n$ & ml) & Group 1 & & oup 2 & & Group 4 & Group 3 \\
\hline & & & & & $\mathrm{HCC}$ & & $\mathrm{H} / \mathrm{LC} \rightarrow \mathrm{I}$ & & No HCC & Suspicious \\
\hline \# subject & & & & & 960 & & & & 183 & 21 \\
\hline Average & & & & & 2362 & & 22 & & 83 & 1241 \\
\hline Median & & & & & 9 & & & & 7 & 8 \\
\hline $\mathrm{SD}$ & & & & & 3161.63 & & 78.19 & & 277.06 & 2384.62 \\
\hline$P$ value & s. Grol & & & & $P<0.0001$ & & $=0.000$ & & & $P<0.024$ \\
\hline
\end{tabular}

Wilcoxon rank sum test. 
interpretation of tumor load, etc.: HCC at study onset (Group 1), developed confirmed HCC during study (Group 2), suspected of possible HCC (Group 3) and no HCC (Group 4) (Tables 1 and 2).

Because we planned 30 month to be the period for follow up of patients for the development of HCC, subjects who had 30 months between the first HCCR-1 positive result and end of study were included which resulted in excluding 42 subjects from the Group 4. Here, 30 months duration doesn't imply the clinical relevance but it is decision duration to observe patients for HCC development. The final number of subjects was 1338 .

None of the subjects had other malignancies or active pulmonary disease. All subjects and controls were subjected to the analysis with individual consent for the study. The use of blood samples was approved by the ethics committee of each 4 institution.

\subsection{Detection of HCCR-1 in serum by sandwich ELISA}

ELISA used in this study is an enzymatically amplified "two-step" sandwich-type immunoassay. The HCCR-1 levels were measured using a sandwich ELISA. The standard curve was constructed with serial dilutions of HCCR-1 $167-360$, ranging from 20 to $320 \mathrm{ng} / \mathrm{ml}$. By using 20, 40, 80, 160, and $320 \mathrm{ng} / \mathrm{ml}$ of HCCR-1 $167-360$ protein, a calibration curve was prepared.

In the assay, standards, controls and unknown serum samples are incubated in microtitration wells which have been coated with anti-HCCR-1 monoclonal antibody (mAb) 54115. After incubation and washing, the wells are treated with another anti-HCCR-1 detection polyclonal antibody labeled with the enzyme horseradish peroxidase (HRP). The reference ranges were obtained by receiver operating characteristics (ROC) curve analyses: $10 \mathrm{ng} / \mathrm{ml}$ was used as a cutoff level for HCC. To determine the serum HCCR-1 cutoff value, ROC analysis was performed using the study population composed of another new group of 880 patients with HCC as a positive control and 380 patients with cirrhosis or chronic hepatitis as a negative control. ROC curve analysis gave a cut-off value of $10 \mathrm{ng} / \mathrm{ml}$ in which sensitivity and specificity were $48.5 \%$ and $93.4 \%$, respectively. With this cut-off value, we then conducted a prospective study for another 1338 patients to evaluate the significance of simultaneous measurement of 2 tumor markers in the diagnosis of HCC (Supplementary Table 1 and Supplementary Fig. 1).

\subsection{Serum AFP concentration}

The AFP level was measured by a 2-step sandwich immunoradiometric assay using a commercially available kit (IRMA AFP MONOCLONAL, BECKMAN COULTER Inc., Fullerton, CA, USA). To determine positive responses, we used the value of $11 \mathrm{ng} / \mathrm{ml}$ as the cutoff for AFP as instructed by the manufacturer.

\subsection{Statistical analysis}

To determine the positive responses, we used $11 \mathrm{ng} / \mathrm{ml}$ as the cutoff value for AFP and $10 \mathrm{ng} / \mathrm{ml}$ for HCCR-1 which was derived from ROC curve analysis from another new 880 patients of HCC as a positive control and 380 patients of liver cirrhosis or chronic hepatitis as a negative control. Serum levels of HCCR1 were presented as the mean \pm SD and compared using Wilcoxon rank sum test and Kruskal-Wallis test. Positive response rates of AFP and HCCR-1 were presented in terms of marginal proportions and compared by $\mathrm{McNemar}$ test for all patients and in each stage of clinicopathologic factors, respectively. A two-tailed significance level of $5 \%$ and SAS (version 9.1) were used for all statistical analyses.

\section{Results}

\subsection{Determination of serum cutoff values}

To determine the cutoff value of HCCR-1, we performed ROC curve analysis using another new 880 patients of HCC and 380 patients of liver cirrhosis or chronic hepatitis. None of the patients had other malignancies or active pulmonary disease. The mean values of serum HCCR-1 in 380 cirrhotic or chronic hepatitis patients and $880 \mathrm{HCC}$ patients were $9.5 \pm 14.4$ and $2436.3 \pm 3300.9 \mathrm{ng} / \mathrm{ml}$, respectively (Table 3 ). There was a statistically significant difference between the mean values of HCCR-1 in HCC patients and liver cirrhosis patients $(P<0.001)$.

ROC curve for liver cirrhosis and HCC patients was obtained and sensitivities, specificities, and total accuracies for five cutoff values were presented (Supplementary Table 1 and Supplementary Fig. 1). We chose the value of $10 \mathrm{ng} / \mathrm{ml}$ as the cutoff for diagnostic evaluation since the specificity is over $90 \%$ at that point although the sensitivity was relatively low. Hereafter, the cutoff value for AFP and HCCR-1 will be $11 \mathrm{ng} / \mathrm{ml}$ and $10 \mathrm{ng} / \mathrm{ml}$, respectively. 
Table 3

The values of serum HCCR-1 in 380 cirrhotic or chronic hepatitis patients and $880 \mathrm{HCC}$ patients for the serum cutoff determination

\begin{tabular}{lrrrcc}
\hline Group & $\mathrm{N}$ & Mean & SD & Range & Median \\
\hline HCC $^{*}$ & 880 & 2436.3 & 3300.9 & $5-11401$ & 9 \\
Liver cirrhosis or chronic hepatitis & 380 & 9.5 & 14.4 & $3-93$ & 6 \\
Total & 1260 & 1704.4 & 2974.7 & $3-11401$ & 8 \\
\hline$* P<0.001$ by Wilcoxon rank sum test. & & & &
\end{tabular}

Table 4

Diagnostic results of HCCR-1 compared with AFP among 1338 patients

\begin{tabular}{llccc}
\hline & \multicolumn{2}{c}{ AFP } & \multicolumn{1}{c}{} \\
\cline { 3 - 4 } & & + & - & Total \\
\hline HCCR-1 & + & 281 & 405 & $686(51.3 \%)^{*}$ \\
& - & 335 & 317 & 652 \\
& Total & $616(46 \%)$ & & $1338(100 \%)$ \\
\hline
\end{tabular}

${ }^{*} P=0.011$ by McNemar test.

Table 5

AFP and HCCR-1 levels related to tumor sizes in HCC patients

\begin{tabular}{llc}
\hline HCC marker & Tumor size & Positive rate \\
\hline AFP & HCC $^{*}$ & $46 \%(616 / 1338)$ \\
& Large HCC $(\geqslant 2 \mathrm{~cm})$ & $66.8 \%(466 / 697)$ \\
& Small HCC ${ }^{\dagger}(<2 \mathrm{~cm})$ & $23.4 \%(150 / 641)$ \\
HCCR-1 & HCC & $51.3 \%(686 / 1338)$ \\
& Large HCC $(\geqslant 2 \mathrm{~cm})$ & $61.5 \%(429 / 697)$ \\
& Small HCC $(<2 \mathrm{~cm})$ & $40.1 \%(257 / 641)$ \\
Combined & HCC & $74.1 \%(990 / 1338)$ \\
$($ AFP + HCCR-1) & Large HCC $(\geqslant 2 \mathrm{~cm})$ & $89.7 \%(625 / 697)$ \\
& Small HCC $(<2 \mathrm{~cm})$ & $56.9 \%(365 / 641)$ \\
\hline
\end{tabular}

*HCC: AFP vs. HCCR-1 ( $p=0.011$ by McNemar test); AFP alone vs. Combined $(P<0.001$ by McNemar test); HCCR-1 alone vs. Combined $(P<0.001$ by McNemar test).

${ }^{\dagger}$ Small HCC: AFP vs. Combined $(P<0.001)$; HCCR-1 vs. Combined $(P<0.001)$.

\subsection{Diagnostic results}

To evaluate whether HCCR-1 could be used as a biomarker for HCC, the serum HCCR-1 level was examined in HCC patients and its association with clinicopathologic characteristics such as tumor size and AFP levels was determined (Tables 2 and 5). Diagnostic results of HCCR-1 compared with AFP are summarized in Table 4. Of 1338 patients, 616 (46\%) were seropositive for AFP, and $686(51.3 \%)$ were sero-positive for HCCR-1 (Table 4). There was a statistical significance between diagnostic efficiencies of AFP (46\%) and HCCR-1 $(51.3 \%)(P=0.011$ by McNemar test) (Table 4) although the diagnostic rate was significantly different in combined analysis of AFP and HCCR-1 for $\mathrm{HCC}(P<0.001)$ (Table 5). The positive rate in collaborative use of AFP and HCCR-1 was increased up to $74.1 \%$ (990 out of 1338 HCC) (Table 5). The cases negative both for AFP and HCCR-1 were 317 (25.9\%). Many cases for AFP-negative HCC were positive for HCCR-1 and vice versa, indicating that the expression of HCCR-1 is not associated with that of AFP in many HCC. The positive rate for HCCR-1 was $56.1 \%$ (405 out of 722) in AFP-negative HCC and the positive rate for AFP was $51.4 \%$ (335 out of 652) in HCCR-1-negative HCC (Table 4).

As a consequence, the serological level of at least one of the two markers was elevated in a majority of HCC patients. These results suggest that the diagnostic rate can be significantly improved through the simultaneous measurement of both HCCR-1 and AFP. Therefore, HCCR-1 could be an useful tumor marker when used together with AFP for diagnosis of HCC.

\subsection{Relationship of HCCR-1 levels with gender, age, hepatitis and countries}

There was no statistical significance in HCCR-1 levels between genders $(P=0.3742)$, ages $(P=0.9412)$, and hepatitis $\mathrm{B}, \mathrm{C}$, or both $(P=0.5290)$. In addition, there was no significant difference in HCCR-1 level between countries $(P=0.2202)$. No correlation was found between serum levels of HCCR-1 and liver functions (AST, ALT, GGT, alkaline phosphatase, albumin, and bilirubin levels in serum, data not shown).

\subsection{Relationship of HCCR-1 levels to tumor size}

Comparison of diagnostic values of total serum AFP and HCCR-1 for HCC is shown in Table 5. Among 697 large $\mathrm{HCC}(\geqslant 2 \mathrm{~cm})$ excluding 641 small HCC out of total $1338 \mathrm{HCC}$, the positive rate of HCCR-1 and AFP was $61.5 \%$ and $66.8 \%$, respectively (Table 5). This shows that the diagnostic rate of HCCR-1 is significantly different from that of AFP in large HCC $(\geqslant$ $2 \mathrm{~cm})(P=0.011$ by $\mathrm{McNemar}$ test $)$.

Importantly, however, the diagnostic rate of HCCR$1(40.1 \%)$ was significantly higher than that of AFP $(23.4 \%)$ in small HCC $(P<0.001)$ (Table 5). This suggests that the diagnostic value of HCCR-1 becomes more evident in small HCC. 
The combined analysis of both AFP and HCCR1 for HCC was performed and it demonstrates that the positive rate for $\mathrm{HCC}$ is increased up to $74.1 \%$ in HCC. In particular, the positive rate for small HCC is increased up to $56.9 \%$. This increase was statistically significant compared to the single analysis of AFP or HCCR-1 alone (Table 5). Therefore, the combined analysis of AFP and HCCR-1 improved the diagnostic rate of HCC both in large and small HCC $(P<0.001)$ (Table 5). This suggests that the simultaneous detection of AFP and HCCR-1 should be effective particularly for the early diagnosis of small HCC.

\section{Discussion}

To determine the possibility of whether HCCR-1 can be used as a biomarker for HCC, the serum HCCR-1 level was examined in HCC patients. Of the $1338 \mathrm{HCC}$ patients, $46 \%$ was sero-positive for AFP, and $51.3 \%$ was sero-positive for HCCR-1. The positive rate in collaborative use of AFP and HCCR-1 was increased up to $74.1 \%$. Elevated HCCR-1 levels were not associated with elevated AFP levels in most cases and the diagnostic efficiency for HCC was similar between AFP and HCCR-1. In other words, many cases for AFP-negative HCC were positive for HCCR-1 and vice versa. The positive rate for HCCR-1 was $56.1 \%$ in AFP-negative HCCs and the positive rate for AFP was $51.4 \%$ in HCCR-1-negative HCCs. As a consequence, the serological level of at least one of the two markers was elevated in a majority of HCC patients.

In small $\mathrm{HCC}(<2 \mathrm{~cm})$, the positive rate of AFP and HCCR-1 was $23.4 \%$ and $40.1 \%$, respectively. The positive rate either for AFP or HCCR-1 was increased up to $56.9 \%$ in small HCC. Therefore the combined analysis of AFP and HCCR-1 improved the diagnostic rate in small HCC. The diagnostic rate of HCCR-1 in small HCC was significantly higher than that of AFP. Our results indicate that the HCCR-1 assay has an advantage over the AFP assay in that it is positive more frequently in patients with early liver cancer.

Worldwide, HCC is the 5th most common cancer and the 3rd most important cause of cancer mortality, with a 5-year survival rate of merely $7 \%[14,15]$. Early detection enables us to provide the patient with the most optimal therapy $[16,17]$. Despite the fact that serum AFP is still the golden standard amongst diagnostic markers for HCC, its diagnostic value is more and more questioned, due to poor sensitivity and specificity of the assay [18]. Previous studies have demonstrated that the sensitivity and specificity of AFP for HCC diagnosis are 41-65 and 80-94\%, respectively, with a cut-off value of $20 \mathrm{ng} / \mathrm{ml}$ [19]. Nevertheless, AFP remains the most commonly used marker in screening and surveillance programs of $\mathrm{HCC}$ to date. Besides its diagnostic value, high AFP level has also been shown to adversely affect the prognosis of patients with HCC treated by hepatic resection or nonsurgical treatment [20,21]. When serum levels are markedly raised $(>400 \mathrm{ng} / \mathrm{ml})$, as occurs in about $50 \%$ of symptomatic cases, it is particularly useful for both diagnosis of HCC and monitoring of treatment response [22,23]. However, AFP is secreted in only about $70 \%$ of HCC, and hence both false-negative and false-positive rates are high with the use of AFP as the serological marker for detection of HCC [2].

Although the HCCR- 1 oncogene was originally identified in human cervical cancer, its serum levels were found to be elevated in patients with HCC but not with cervical cancer, implying its close relationship with hepatocarcinogenesis [7]. Furthermore, various studies demonstrated that HCCR-1 could be used as a serological test for the diagnosis of patients with HCC [2,7-10]. Although we previously developed HCCR-1 indirect ELISA assay for serological test [7], the development of sandwich ELISA assay was essential to resolve the specificity issue. To achieve this goal, we developed HCCR-1 sandwich ELISA assay in this study to improve the serological assay using HCCR-1 for HCC. The HCCR-1 sandwich ELISA assay revealed that elevated HCCR-1 levels are associated with an increase in the risk of developing HCC.

In conclusion, diagnostic rate of HCC can be improved through the simultaneous measurement of both HCCR-1 and AFP. HCCR-1 could be used as a supplementary to AFP in the diagnosis of HCC, but each of them has no satisfactory accuracy in detecting HCC or prefiguring the prognosis when used alone. Given the high heterogeneity of HCC, it is currently thought that an optimal serological test for HCC will be based on the simultaneous measurement of two or three highly specific serological markers. Combining the information from AFP and HCCR-1 increases the diagnostic rate over AFP alone in particular in small HCC and may discover the recurrence of HCC at earlier period. Our results indicate that the HCCR-1 assay has an advantage over the AFP assay since its level is increased according to disease progression and it is positive more frequently in patients with early liver cancer. 


\section{Acknowledgements}

This study was supported by the 'Seoul R\&BD Program' 2006. Also supported by a grant of the Korea Health 21 R\&D Project, Ministry of Health \& Welfare, Republic of Korea (02-PJ10-PG6-AG01-0003). These authors (Guoxin Zhang., Seon-Ah Ha. and Hyun Kee Kim) contributed equally to this work.

\section{Supplemental material}

To obtain the supplemental Table and Figure, please contact the corresponding author Prof. Jin Woo Kim: jinwoo@catholic.ac.kr.

\section{Conflict of interest}

The authors declare that they have no competing interests.

\section{References}

[1] D.M. Parkin, F. Bray, J. Ferlay and P. Pisani, Estimating the world cancer burden: Globocan 2000, Int J Cancer 94 (2001), 153-156.

[2] L. Zhou, J. Liu and F. Luo, Serum tumor markers for detection of hepatocellular carcinoma. World J Gastroenterol 12(8) (2006), 1175-1181.

[3] J. Bruix, M. Sherman, J.M. Llovet et al., EASL Panel of Experts on HCC: Clinical management of hepatocellular carcinoma. Conclusions of the Barcelona-2000 EASL conference. European Association for the Study of the Liver, J Hepatol 35 (2001), 421-430

[4] J.C. Sheu, J.L. Sung, D.S. Chen et al., Early detection of hepatocellular carcinoma by real-time ultrasonography. A prospective study, Cancer 56 (1985) 660-666.

[5] P. Stefaniuk, J. Cianciara and A. Wiercinska-Drapalo, Present and future possibilities for early diagnosis of hepatocellular carcinoma, World J Gastroenterol 16(4) (2010), 418-424.

[6] M. Soresi, C. Magliarisi, P. Campagna et al., Usefulness of alpha-fetoprotein in the diagnosis of hepatocellular carcinoma, Anticancer Res 23 (2003), 1747-1753.

[7] S.K. Yoon, N.K. Lim, S-A. Ha et al., The human cervical cancer oncogene protein is a biomarker for human hepatocellular carcinoma, Cancer Res 64 (2004), 5434-5441.

[8] D. Geng, J. Chen and L. Chen, Effect of matrine on expression of HCCR-1 and HCCR-2 proteins in cultural human hepatocellular carcinomas cells, Chinese J Cancer Res 17(4) (2005), 265-269.

[9] J.B. Xia, Z.H. Zhang, Y.J. Tian et al., Cloning, prokaryotic expression and polyclonal antibody preparation of HCCR-1: a new biomarker for hepatocellular carcinoma, Zhonghua Gan Zang Bing Za Zhi 14(2) (2006), 101-104.

[10] Y. Yang, G.X. Zhang, R.H. Shi et al., Overexpression of HCCR-1 in hepatocellular carcinoma cells and its clinical significance, Zhonghua Gan Zang Bing Za Zhi 15(3) (2007), 223-224.

[11] J Ko, Y.H. Lee, S.Y. Hwang et al., Identification and differential expression of novel human cervical cancer oncogene HCCR-2 in human cancers and its involvement in p53 stabilization, Oncogene 22 (2003), 4679-4689.

[12] J. Ko, S.M. Shin, Y.M. Oh et al., Transgenic mouse model for breast cancer: induction of breast cancer in novel oncogene HCCR-2 transgenic mice, Oncogene 23 (2004), 1950-1953.

[13] G.W. Cho, S.M. Shin, H. Namkoong et al., The phosphatidylinositol 3-kinase/Akt pathway regulates the HCCR-1 oncogene expression, Gene 384 (2006), 18-26.

[14] F.X. Bosch, J. Ribes, R. Cleries and M. Diaz, Epidemiology of hepatocellular carcinoma, Clin Liver Dis 9 (2005), 191-211.

[15] C. Verhoef, O. Visser, R.A. de Man et al., Hepatocellular carcinoma in the Netherlands incidence, treatment and survival patterns, Eur J Cancer 40 (2004), 1530-1538.

[16] M.A. Avila, C. Berasain, B. Sangro and J. Prieto, New therapies for hepatocellular carcinoma, Oncogene 25 (2006), 38663884.

[17] P.J. Johnson, R. Williams, H. Thomas et al., Induction of remission in hepatocellular carcinoma with doxorubicin, Lancet 1 (1978), 1006-1009.

[18] G.I. Abelev, S.D. Perova, N.I. Khramkova et al., Production of embryonal alpha-globulin by transplantable mouse hepatomas, Transplantation 1 (1963), 174-180.

[19] S. Gupta, S. Bent and J. Kohlwes, Test characteristics of alphafetoprotein for detecting hepatocellular carcinoma in patients with hepatitis C. A systematic review and critical analysis, Ann Intern Med 139 (2003), 46-50.

[20] C.N. Yeh, W.C. Lee and M.F. Chen, Hepatic resection and prognosis for patients with hepatocellular carcinoma larger than $10 \mathrm{~cm}$ : two decades of experience at Chang Gung memorial hospital, Ann Surg Oncol 10 (2003), 1070-1076.

[21] C.B. O’Suilleabhain, R.T. Poon, J.L. Yong et al., Factors predictive of 5-year survival after transarterial chemoembolization for inoperable hepatocellular carcinoma, Br J Surg 90 (2003), 325-331.

[22] P.J. Johnson and R. Williams, Serum alpha-fetoprotein estimations and doubling time in hepatocellular carcinoma: Influence of therapy and possible value in early detection, $J$ Nat Cancer Inst 64 (1980), 1329-1332.

[23] P.J. Johnson, The Role of Serum Alpha-fetoprotein Estimation in the Diagnosis and Management of Hepatocellular Carcinoma, in: Clinics in Liver Disease, A. Di Bisceglie, ed., WB Saunders, Philadelphia, 2001, pp. 145-159. 


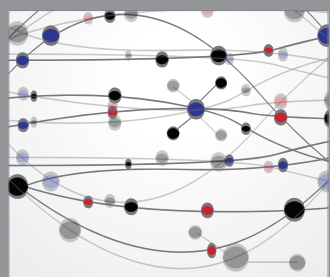

The Scientific World Journal
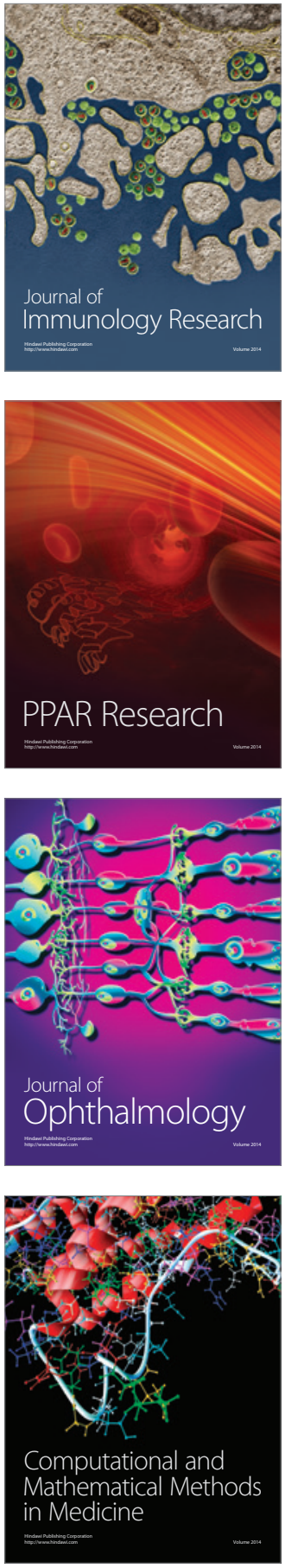

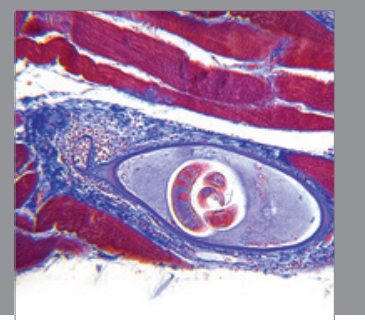

Gastroenterology

Research and Practice
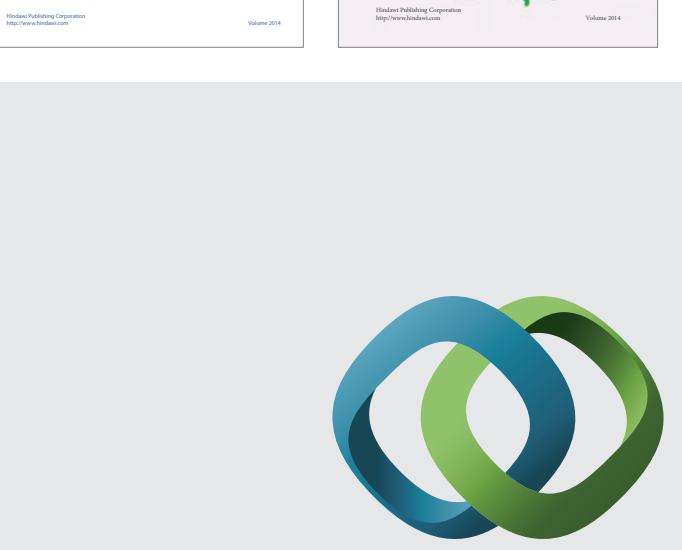

\section{Hindawi}

Submit your manuscripts at

http://www.hindawi.com
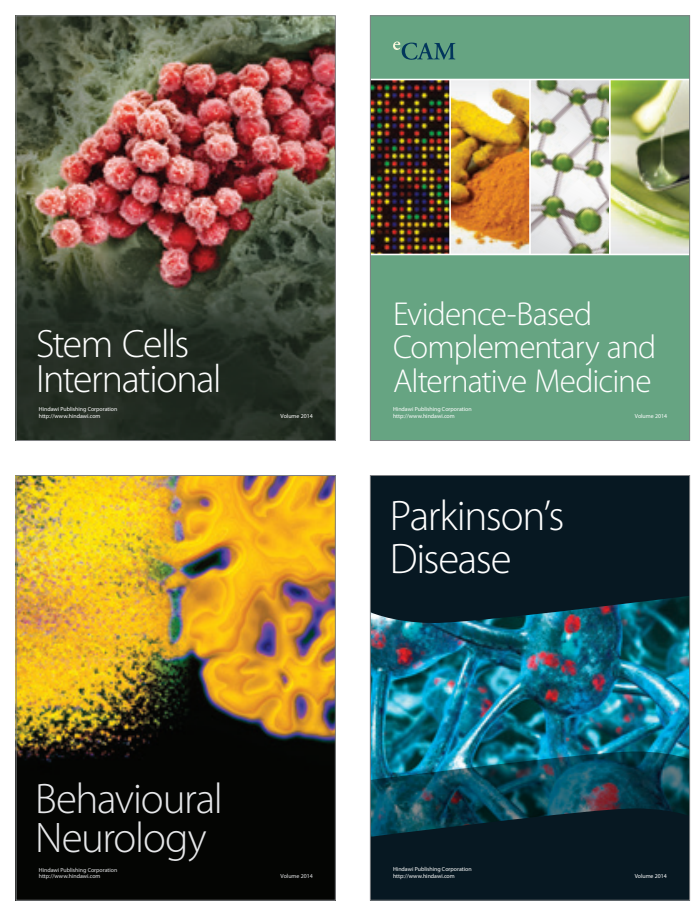

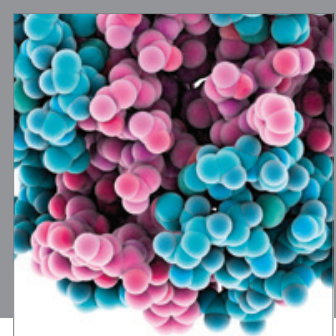

Journal of
Diabetes Research

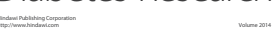

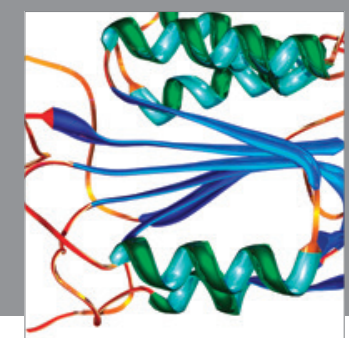

Disease Markers
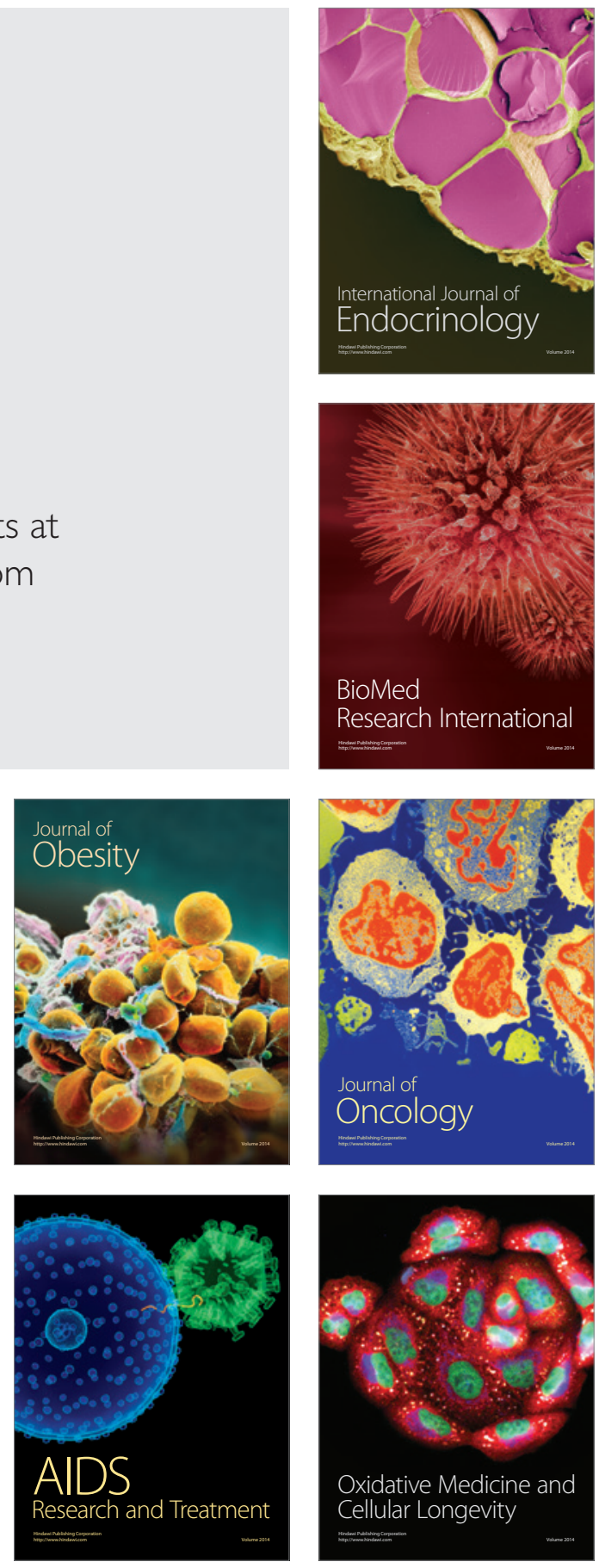